\title{
Customised, individually made total knee arthroplasty shows promising 1-year clinical and patient reported outcomes
}

\author{
Céline S. Moret ${ }^{1} \cdot$ Michael T. Hirschmann ${ }^{1} \odot \cdot$ Nicole Vogel $^{2} \cdot$ Markus P. Arnold $^{2}$
}

Received: 7 March 2021 / Accepted: 1 July 2021 / Published online: 16 July 2021

(c) The Author(s) 2021

\begin{abstract}
Introduction Customised individually made (CIM) implants for total knee arthroplasty (TKA) were introduced about 10 years ago. These implants aim to reduce the risk of prosthesis-related issues resulting from anthropometric differences between different knees.

The purpose of this study was to analyse the short-term clinical outcome and patient reported outcome measures (PROMs) of a specific CIM implant, the ORIGIN ${ }^{\circledR}$ knee replacement system (Symbios, Yverdon-les-Bains, Switzerland), which was introduced in 2018.

Materials and methods This is a prospective cohort study of patients undergoing primary posterior-stabilised (PS) CIM TKA using the specific ORIGIN ${ }^{\circledR}$ knee replacement system, (Symbios, Yverdon-les-Bains, Switzerland). TKAs were performed from February 2019 to October 2020. Data was collected preoperatively and postoperatively at 4 and 12 months. Outcome measures included the objective part of the Knee Society Score (KSS) with the range of motion (ROM) and the following PROMs: the Knee injury and Osteoarthritis Outcome Score (KOOS), the Forgotten Joint Score (FJS-12), the EuroQol, five dimensions, three levels (EQ-5D-3L) with the EuroQol visual analogue scale (EQ-VAS) and patient satisfaction. Differences in pre- to preoperative data were assessed with paired sample $t$ tests. A $p$ value $<0.05$ was considered significant.

Results Twenty-five CIM TKA (20 patients, 8 female) were included. The mean age at surgery was 66 years (SD, 6.9). At 4 and 12 months, significant improvements in the KSS $(p<0.001)$, the ROM $(p<0.001)$, all KOOS subscales $(p<0.001)$, the FJS $(p<0.001)$ and the EQ-5D-3L $(p<0.026)$ were found. Satisfaction rate was $91 \%$ and $88 \%$ at 4 and 12 months, respectively. Intraoperative complications did not occur and no revision surgeries were undertaken.

Conclusions The present study demonstrated significant improvements in the KSS and specific PROMs 1 year after CIM TKA. This study suggests that CIM TKA is a safe and suitable option, which can yield good clinical outcome and PROMs at least during short-term follow-up.
\end{abstract}

Keywords Total knee arthroplasty $\cdot$ Customised individually made $\cdot$ patient-specific implants $\cdot$ Knee Society Score $\cdot$ Patient reported outcome measures $\cdot$ Satisfaction

Céline S. Moret and Michael T. Hirschmann contributed equally.

Michael T. Hirschmann

michael.hirschmann@unibas.ch

Céline S. Moret

celinesaphena.moret@unibas.ch

Nicole Vogel

vogel.leonardo-ortho@hin.ch

Markus P. Arnold

arnold.leonardo-ortho@hin.ch
1 Department of Orthopaedic Surgery and Traumatology, University of Basel, Kantonsspital Baselland, 4101 Bruderholz, Switzerland

2 Practice Leonardo, Hirslanden Klinik Birshof, Reinacherstrasse 28, 4142 Münchenstein, Switzerland 


\section{Introduction}

About $20 \%$ of patients are unsatisfied after primary total knee arthroplasty (TKA). Patients report persisting pain, instability, stiffness or a persistent or recurrent effusion requiring a subsequent revision [1-8]. In particular, aseptic loosening, instability and patellofemoral disorders, which are responsible for about $40 \%$ of all revision causes, are known to be affected by TKA component size or positioning $[6,7]$. These issues might theoretically be reduced by a more individualised, patient-specific approach. Customised individually made (CIM) TKA requires patient-specific implants and instrumentation to better fit the individual anthropometric knee joint characteristics.

Hirschmann et al. [8-10] have shown a high variability of the medial femoral mechanical angle (mFMA) and the medial tibial mechanical angle (mTMA) within the three classical limb phenotypes in a non-osteoarthritic Caucasian population. They recommended that the overall coronal lower limb alignment should not only be classified in neutral, varus or valgus on the basis of the hip-knee-ankle angle (HKA), but that femoral and tibial joint lines should be considered as well. Furthermore, several studies have demonstrated that among different ethnic groups different mediolateral-to-anteroposterior ratio of tibia and femur exist [11-13].

Conventional, off-the-shelf (OTS) implants are based on anthropometric measurements of a defined standard, however, mostly Caucasian population [11]. Although multiple models and sizes of OTS implants exist, it can be challenging to find the most adapted for the patient's knee morphology. The surgeons' experience with different implant models or the availability in a specific hospital can also play a role in the choice of the implant. Thus, quite recently developed CIM implants are specifically adapted to the individual knee morphology, especially in patients who present less conventional anthropometric characteristics. Hence, the aim of this study was to analyse the clinical outcome and specifically patient reported outcome measures (PROMs) after TKA with a specific CIM implant. It was hypothesised that CIM TKA shows good short-term clinical and patient reported outcomes.

\section{Materials and methods}

\section{Study design and population}

This study was approved by the local ethics committee (reference: 2016-01777) and written informed consent was obtained from all patients willing to participate.
This is a single-site, prospective cohort study including patients undergoing primary posterior-stabilised (PS) CIM TKA using the ORIGIN ${ }^{\circledR}$ knee replacement system (Symbios, Yverdon-les-Bains, Switzerland). Routinely, all patients scheduled for a TKA were asked to complete PROMs before and after the surgery. The CIM TKAs were performed between February 2019 and October 2020 in a private hospital by one experienced board-certified surgeon specialised in knee surgery (MPA).

The decision whether to implant the ConforMIS ${ }^{\circledR}$ or the ORIGIN ${ }^{\circledR}$ CIM knee replacement system depended on the integrity of the posterior cruciate ligament (PCL) and on the preoperative knee flexion. In patients with intact PCL the cruciate-retaining (CR) version of the ConforMIS ${ }^{\circledR}$ knee replacement system was opted for whereas patients with either preoperative or expected intraoperative PCL insufficiency or passive flexion of less than $110^{\circ}$ underwent CIM TKA with the ORIGIN ${ }^{\circledR}$ PS knee replacement system. Inclusion criteria for the ORIGIN ${ }^{\circledR}$ CIM TKA were primary TKA, non-inflammatory degenerative or inflammatory disease, less than $10^{\circ}$ of recurvatum, a coronal varus or valgus HKA deviation of less than $10^{\circ}$, a pre-existing PCL lesion or the presence of large posterior osteophytes, which would lead to a PCL insufficiency after their removal, an absence of collateral ligament distention and/or excessive extra-articular deformation. Patients with insufficient knowledge of German, English, French or Italian were excluded.

Between February 2019 and October 2020, 35 CIM TKAs (29 patients, 12 female) were performed. Of those, 25 CIM TKAs (20 patients, 8 female) had available PROMs and were thus included in this study. Five patients had a bilateral CIM TKA and three had a contralateral TKA other than CIM TKA. The mean age at surgery was 66 years (SD, 6.9). Patient demographics are described in Table 1.

Table 1 Patient demographics

\begin{tabular}{lll}
\hline Variables at surgery & & CIM TKA $(n=25)$ \\
\hline Age, mean (SD) & & 66 years $(6.9)$ \\
Body mass index, mean (SD) & & $28.1 \mathrm{~kg} / \mathrm{m}^{2}(5.5)$ \\
Sex, $n(\%)$ & Male & $16(64)$ \\
& Female & $9(36)$ \\
Side, $n(\%)$ & Right & $12(48)$ \\
& Left & $13(52)$ \\
Kellgren-Lawrence score, $n(\%)$ & 3 & $2(8)$ \\
& 4 & $23(92)$ \\
Alignment, $n(\%)$ & Neutral & $4(16)$ \\
& Varus & $17(68)$ \\
& Valgus & $4(16)$ \\
\hline
\end{tabular}

CIM TKA customised, individually made total knee arthroplasty, $S D$ standard deviation 


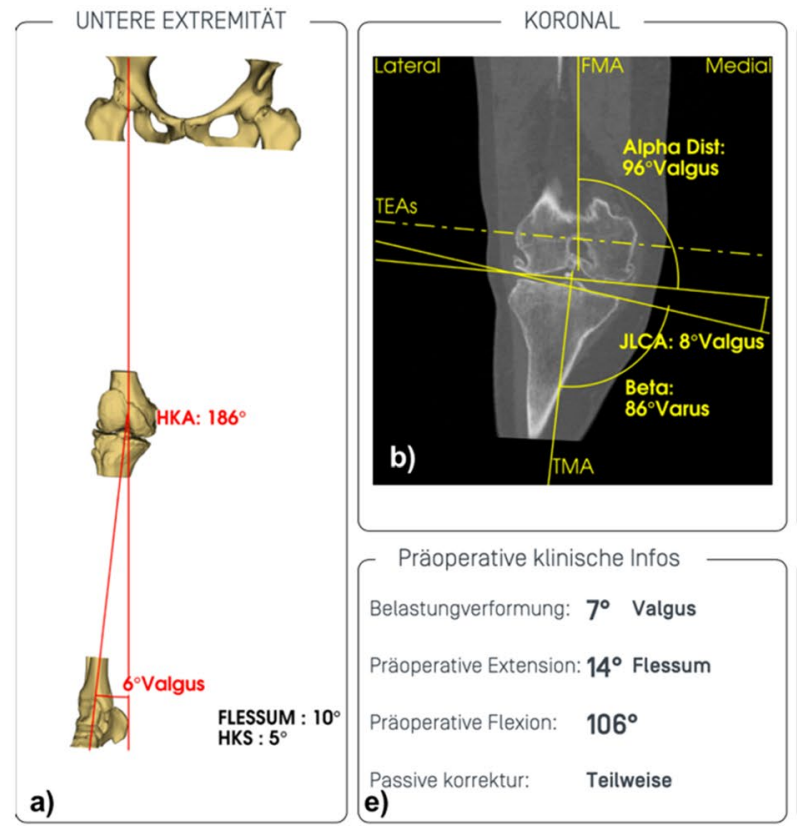

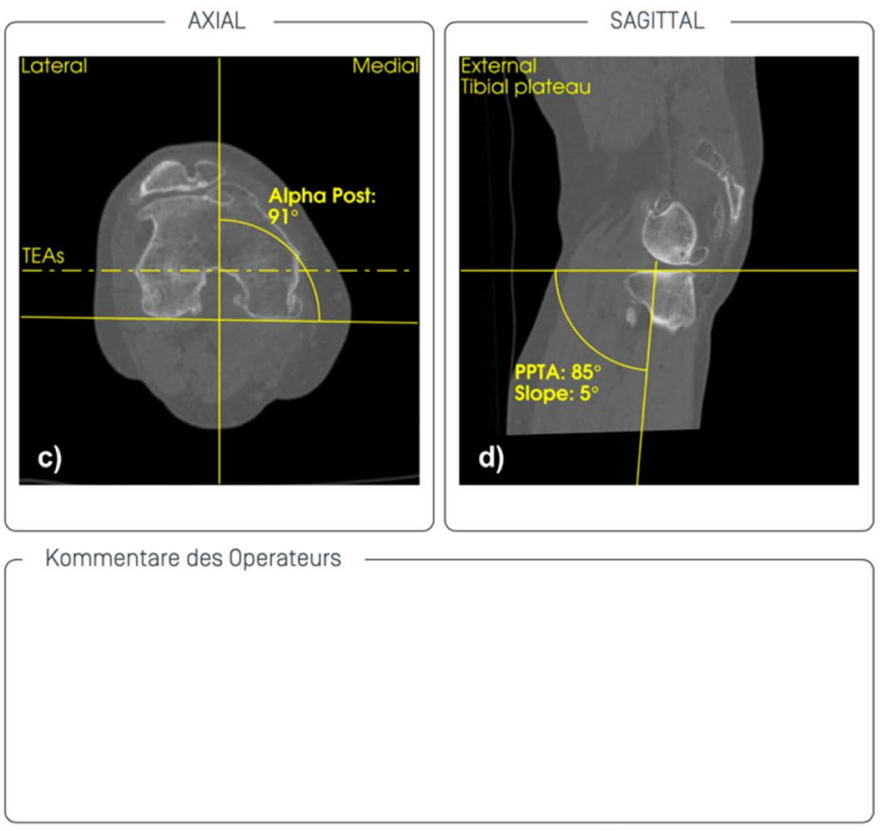

symbios
Fig. 1 Example of preoperative analysis in a female patient with valgus osteoarthritis of her right knee (KNEE-PLAN ${ }^{\circledR}$, Symbios). a Determination of the limb alignment with the hip-kneeankle angle (HKA) and the hip-knee-shaft angle (HKS) in the coronal plane. b Determination of the mechanical medial distal femoral angle (Alpha Dist), the mechanical medial proximal tibial angle (Beta), and the joint line convergence angle (JLCA) in the coronal plane. The femo-

\section{Implant design, production process and surgical technique}

The implant is based on the preoperative computed tomography of the osteoarthritic knee, according to the Imperial Knee Protocol, and on its subsequent three-dimensional (3D) reconstruction [14]. The alignment and morphology of the knee are analysed measuring different angles and axis using a specialised planning software by the manufacturer's engineer (Knee-Plan ${ }^{\circledR}$, Symbios, Yverdon-les-Bains, Switzerland) (Fig. 1).

After assessment of cartilage wear, subchondral bone loss and location of osteophytes of the femur and tibia, the engineer determines in a best-fit scenario the individual femoral J-curves. The ORIGIN ${ }^{\circledR}$ realignment strategy is a personalised postoperative alignment according to the restricted phenotype alignment protocol, which aims to reproduce the individual coronal knee phenotype in the limits of a safe target zone [15]. The tibial baseplate is asymmetric to fit the native tibial plateau and the angle of the tibia keel is adapted to the metaphysis [16]. The design and fitting of the components are reproduced with a 3D software (SolidWorks ${ }^{\circledR}$ ral mechanical angle (FMA), the tibial mechanical angle (TMA) and the surgical transepicondylar axis (TEAs) are marked as reference. c Determination of the rotational alignment (Alpha post) of the distal femur in the axial plane. In this case, the posterior condylar angle is $1^{\circ}$. d Determination of the posterior proximal tibial angle (PPTA) in the sagittal plane. In this case, the posterior slope is $5^{\circ}$. e Clinical information provided by the surgeon

software, Dassault Systèmes, Vélizy-Villacoublay, France) [16]. This allows a reproduction of asymmetries, an optimal coverage of the resected bone surface by the implant and an anteroposterior (AP) to mediolateral (ML) ratio adapted to each patient. In addition, ligament balancing is improved due to the reproduction of the patient's pre-osteoarthritic anatomy [16]. Thus, resection laxity due to asymmetric bone cuts can be avoided [16].

After validation of the planning summary (Fig. 2) by the surgeon, the production of the CIM knee system is undertaken. It comprises a customised PS tibial and femoral component, with or without patellar component, and individually printed custom-cutting-blocks for patientspecific instrumentation. Anteroposterior stability relies on the ultra-congruent polyethylene for the first $60^{\circ}$ of flexion, then the post-cam system engages [16]. The prosthesis has a chrome-cobalt-molybdenum ( $\mathrm{CrCoMo}$ ) femoral and a titanium alloy (Ti6Al4V) tibial component with a snap fit, fixed bearing, ultra-congruent, ultrahigh molecular weight polyethylene (UHMWPE) insert. Both components are cemented.

The CIM TKA was usually performed through a medial parapatellar approach with individually printed 


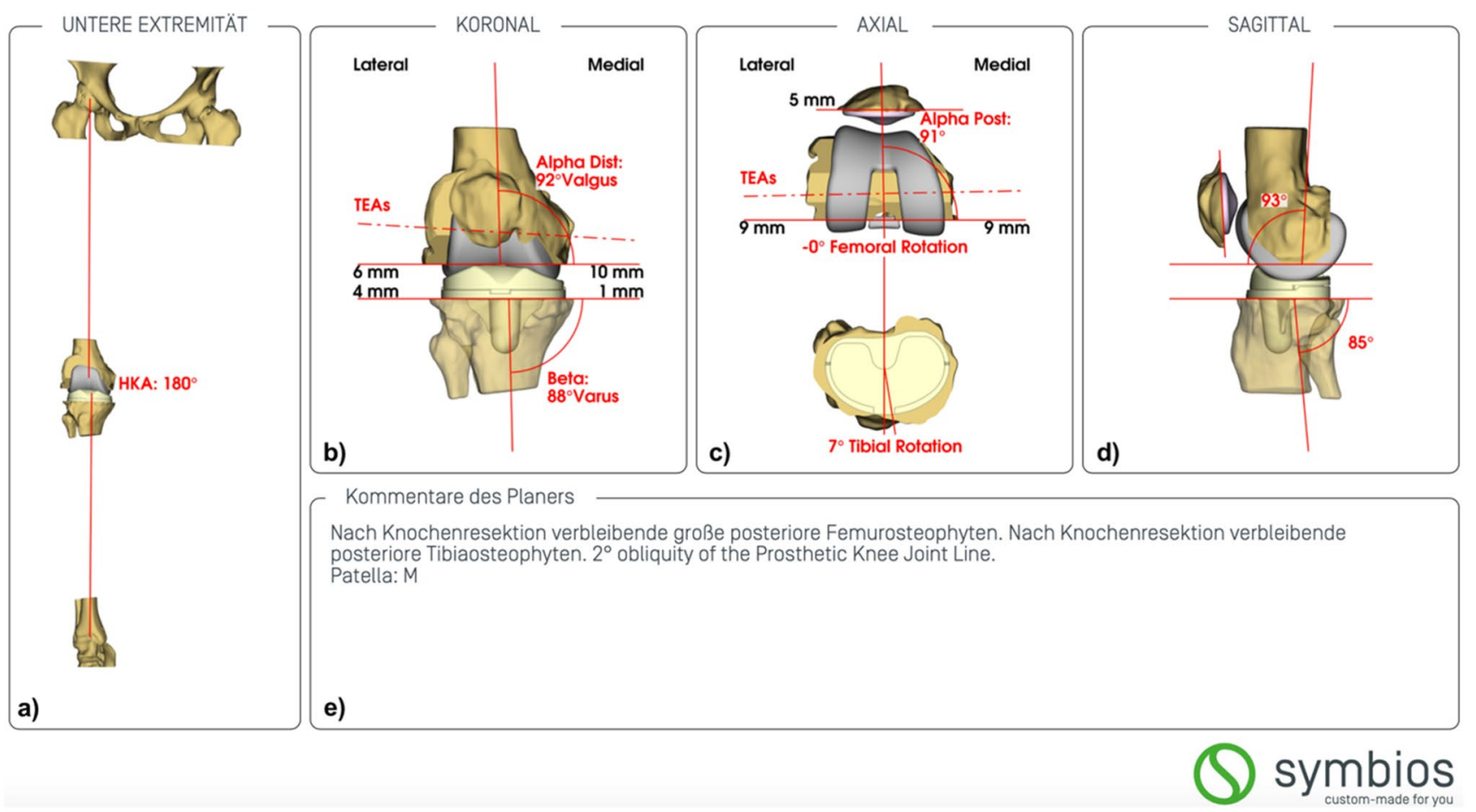

Fig. 2 Example of the planning summary suggested for the same patient as seen in Fig. 1. a Restoration of the limb alignment to a hip-knee ankle angle (HKA) of $180^{\circ}$ in this case. b Restoration to a mechanical medial distal femoral angle (Alpha Dist) and mechani-

custom-cutting-blocks and custom trials. In tight valgus knees, a lateral approach with osteotomy of the tibial tuberosity was preferred [17]. For optimal positioning and orientation of the cutting blocks, the surgeon aimed for a best-fit to the bone that ensured optimal stability of the four cutting block zones resting on the bone as presented on the provided three-dimensional model of the femur and tibia. The varus or valgus alignment of the tibial cutting block was assessed with an extramedullary reference rod which helped to determine if a slight correction before resection was required.

All patients received the same standard postoperative follow-up and physical therapy.

\section{Clinical outcome and PROMs}

The following parameters were assessed preoperatively and postoperatively after four and 12 months during routine follow-up consultations according to the publication by Vogel et al. [18]. The surgeon assessed the objective part of the Knee Society Score (KSS), which measures pain, range of motion (ROM), stability and alignment. Its score varies between 0 and 100 [19].

Following PROMs were completed: (1) the Knee injury and Osteoarthritis Outcome Score (KOOS), (2) the Forgotten cal medial proximal tibial angle (Beta) of $92^{\circ}$ and $88^{\circ}$ in this case, respectively. c Axial rotational alignment of the femoral component and tibial component. d Sagittal alignment of the femoral and tibial component. e Comments from the engineer

Joint Score (FJS-12) and (3) the EuroQol five dimensions, three levels (EQ-5D-3L). The KOOS consists of five subscales and captures pain, symptoms, activities of daily living (ADL), sport and recreational activities and knee-related quality of life (QoL). Each subscale has a score ranging from 0 to 100 [20]. The Forgotten Joint Score assesses joint awareness with a total score from 0 to 100 points [21]. The EQ-5D-3L evaluates health-related quality of life with an index score ranging from 0 to 1 and a and visual analogue scale (EQ-VAS) ranging from 0 to 100 points [22].

In the KSS and PROMs, a higher total score indicates a better outcome.

Moreover, patients' satisfaction with the surgery was evaluated on a five-point Likert scale ranging from "very satisfied" to "very unsatisfied". Patient's willingness to undergo CIM TKA again (yes/no) was also assessed during follow-up after 12 months.

Intraoperative complications and revision surgeries were also recorded.

All study data were collected and managed with the Research Electronic Data Capture (REDCap). 


\section{Statistical method}

Statistical analysis was performed with IBM SPSS Statistics for Windows version 27 (IBM Corp., Armonk, NY, US). Descriptive statistics are presented with mean and standard deviation (SD) for continuous variables, frequency counts and percentages for categorical variables. Normal distribution was confirmed with the Kolmogorov-Smirnov test and the paired sample $t$ test was applied to determine pre- to postoperative differences of continuous variables. Results are presented with a $95 \%$ confidence interval $(\mathrm{CI})$ and a $p$ value of $<0.05$ was considered significant.

\section{Results}

The data of 25 and 16 CIM TKA were analysed at 4 and 12 months, respectively. There were no losses to follow-up. A neutral and a varus limb alignment were attained in 24 and 1 CIM TKA, respectively. There were significant preto postoperative improvements in KSS $(p<0.001)$, ROM $(p<0.001)$, all KOOS subscales $(p<0.001)$, FJS $(p<0.001)$ and EQ-5D-3L $(p<0.026)$ at both follow-up consultations. The EQ-VAS improved nonsignificantly. Detailed results can be seen in Table 2 .

At 4 and 12 months, $91 \%$ and $88 \%$ of patients reported to be very satisfied or satisfied with their knee implant, respectively, whereas the remaining patients were neither satisfied nor dissatisfied. After 12 months, all patients would undergo the same surgery again.

The patient-specific instrumentation (PSI) fitted seamlessly to the bony morphology. The pins were stable and no repositioning was necessary. No other intraoperative complications or revision surgeries during the follow-up period occurred.

\section{Discussion}

The most important finding of the present study is the safety and reliability of the ORIGIN $^{\circledR}$ CIM knee replacement system, which shows good short-term clinical and patient reported outcomes.

There are currently no available publications assessing non-radiological clinical and patient reported outcomes after CIM TKAs with the ORIGIN ${ }^{\circledR}$ knee replacement system. Hence, a comparison can only be undertaken with results of the CIM ConforMIS ${ }^{\circledR}$ knee replacement system. The present study demonstrated, as expected, a significant pre- to postoperative improvement in the objective part of the KSS at 4 and 12 months. This improvement is comparable to the difference of 38.3 points (SD, 14.4) found at 3 months by Wheatley et al. [23]. The same author, however, detected no significant differences in the objective part of the KSS between the CIM and the OTS TKA groups [23]. Reimann et al. [24] only found a significant increase in the function score (subjective part) of the KSS, thus leading to a significantly better entire KSS, which the authors attribute to a younger mean age of 65 years in their CIM TKA cohort, whereas White and Ranawat [25], who compared 74 patient knees 2 years after undergoing TKA with different implants (CIM TKA:21/OTS TKA:11/PS OTS TKA:42) even found worse scores in the objective and subjective parts of the

Table 2 Clinical outcome and PROMs at different time-points

\begin{tabular}{|c|c|c|c|c|c|}
\hline \multirow[t]{3}{*}{ Measurement } & \multicolumn{5}{|l|}{ CIM TKA } \\
\hline & \multirow{2}{*}{$\begin{array}{l}\text { Preoperative } \\
n=25 \\
\text { Mean (SD) }\end{array}$} & \multicolumn{2}{|c|}{ 4-month follow-up } & \multicolumn{2}{|c|}{ 12-month follow-up } \\
\hline & & $\begin{array}{l}n=25 \\
\text { Mean (SD) }\end{array}$ & $\begin{array}{l}\text { Difference to preoperative } \\
p \text { value }(95 \% \mathrm{CI})\end{array}$ & $\begin{array}{l}n=16 \\
\text { Mean (SD) }\end{array}$ & $\begin{array}{l}\text { Difference to preoperative } \\
p \text { value }(95 \% \mathrm{CI})\end{array}$ \\
\hline oKSS & $46(13.9)$ & $90(6.1)$ & $<0.001(37.2-50.8)$ & $94(5.8)$ & $<0.001(38.3-56.9)$ \\
\hline ROM & 109 (16.7) & $125(5.7)$ & $<0.001(7.8-19.6)$ & $129(5.3)$ & $<0.001(11.9-28.1)$ \\
\hline KOOS symptoms & $44(12.9)$ & $68(13.7)$ & $<0.001(13.7-33.7)$ & $80(8.5)$ & $<0.001(34.1-47.9)$ \\
\hline KOOS pain & $50(14.3)$ & $75(12.6)$ & $<0.001(16.4-33.5)$ & $86(11.4)$ & $<0.001(25.9-47.5)$ \\
\hline KOOS ADL & $60(16.9)$ & $79(14.7)$ & $<0.001(9.3-29.2)$ & $87(10.5)$ & $<0.001(16.8-34.8)$ \\
\hline KOOS sports & $26(18.8)$ & $56(25.1)$ & $<0.001(16.3-41.0)$ & $64(30.8)$ & $<0.001(26.9-58.5)$ \\
\hline KOOS QoL & $33(15.9)$ & $58(25.1)$ & $<0.001(14.2-35.8)$ & $70(20.3)$ & $<0.001(25.0-51.3)$ \\
\hline FJS-12 & $22(14.7)$ & $52(23.7)$ & $<0.001(19.1-42.3)$ & $73(19.7)$ & $<0.001(40.7-63.5)$ \\
\hline EQ-5D-3L & $0.707(0.16)$ & $0.824(0.16)$ & $<0.026(0.155-0.221)$ & $0.914(0.11)$ & $0.001(0.105-0.308)$ \\
\hline EQ-VAS & $75(16.9)$ & $78(17.6)$ & $0.548(-7.4$ to 13.5$)$ & $84(10.5)$ & $0.164(-3.7$ to 20.1$)$ \\
\hline
\end{tabular}

oKSS objective part of the Knee Society Score, ROM range of motion, KOOS Knee injury and Osteoarthritis Outcome Score, ADLactivities of daily living, $Q o L$ quality of life, FJS-12 Forgotten Knee Joint Score, EQ-5D-3LEuroQol five dimensions three levels, VAS visual analogue scale, $S D$ standard deviation, CI confidence interval 
KSS as well as lower satisfaction rates in patients with CIM implants.

Concerning the ROM, Schwarzkopf et al. [26], analysing 621 TKAs (CIM implants:307/OTS implants:314), measured a decrease of $3.44^{\circ} 1$ year postoperatively compared to preoperatively in the CIM TKA groups; a result, the authors considered without any clinical significance due to their nonidentical ROM measurement protocol. Moreover, comparison of the ROM between the two groups at a minimum of 1 year, did not show a significant difference [24-26].

Almost one-third of patients required manipulation under anaesthesia (MUA) to improve the ROM to a mean of $115^{\circ}$ at 2 years in the CIM TKA cohort of White et Ranawat [25]. These findings are not in line with the present study, showing no MUA or revision and a mean ROM of $125^{\circ}$ and $129^{\circ}$ at 4 and 12 months, respectively.

Potential stiffness leading to a reduced ROM might be explained with the limited options a surgeon has at hand intraoperatively in terms of resection depth. As only two inlay heights $(0$ and $+2 \mathrm{~mm})$ are available, some surgeons tend to underresect and thus render the knee rather tight in extension and flexion. This is not a problem related to the CIM implant itself, but to the PSI. There is well-established evidence about the accuracy and limitations of PSI with regards to the implant orientation [27-30]. When assessing the coronal alignment of the tibial component, Zahn et al. [31] observed more outliers from the neutral mechanical medial proximal tibial angle in the PSI group than in groups comprising extramedullary or intramedullary implant positioning technique or computer-navigated implantation. From experience, ConforMIS ${ }^{\circledR}$ PSI for the tibial component have a tendency to tilt downward when fixed to the tibia, hence recreating less posterior slope than planned. This occurs less frequently with the ORIGIN ${ }^{\circledR}$ PSI, as the ORIGIN $^{\circledR}$ tibial guide uses different and more reliable reference points.

Concerning PROMs, Reimann et al. [24] found no significant differences between the CIM and OTS TKA group 2-3 years after surgery in all KOOS subscales. This shows that complex everyday activities (e.g. getting in the bath, gardening or getting out of the car) still remain challenging after a TKA independently of the utilization of CIM or OTS implants [24]. In the present study, the lowest mean score of 64 points was attained in the subscale sports. This result builds on the data from Reimann et al. [24] who found also lower mean scores ( $<60$ points) in the same subscale for both CIM TKA and OTS TKA groups, explained by the apprehension or refusal of many patients to run, jump or kneel after TKA. The present study showed a significant mean pre- to postoperative improvement in the FJS-12 with a mean score of 73 points (SD 19.7) at 12 months. This result is within the range of 67.6 (SD 27.8) to 82 (range, 70-94) found for OTS implants in different publications [32-34]. Similarly, Wheatley et al. [23], revealed no significant difference in the FJS-12 between patients who underwent CIM or OTS TKAs at a mean of 2 years postoperatively.

Patient satisfaction after CIM TKA was higher at 4 months (91\% vs $83 \%$ ) and slightly lower at 12 months ( $88 \%$ vs $91 \%$ ) compared to a publication about OTS TKA [35]. This slightly worse result at 1 year might be due to the fact that potentially satisfied and very satisfied patients have not yet attended the 12-month follow-up. Nevertheless, satisfaction rate of the included patients is well above patient satisfaction of approximately $80 \%$ recorded in multiple other studies [1-8]. Despite the lack of long-term evidence that CIM TKA directly improves clinical and patient outcomes, Reimann et al. [24], who compared patient satisfaction of 84 CIM TKAs with 57 OTS TKAs 2-3 years after surgery, found that global satisfaction was significantly better in the CIM TKA group. This result might be partially attributed to a placebo effect since patients are aware that they have received the latest generation of TKA implants.

Recent articles have shown that anteroposterior to mediolateral femoral and tibial ratios differ between ethnic groups and that component overhang might lead to knee pain [11-13, 36]. CIM implants provide better cortical bone coverage and thus reduce the risk of overhang and undercoverage [37]. Indeed, Klasan et al. [38] demonstrated that overhang of the tibial component decreased the clinical outcome in terms of KOOS by the same margin as loss of $16 \%$ of coverage. Furthermore, optimal bone coverage could lower the risk of bleeding from resected bone surfaces, reducing postoperative knee swelling as well as potentially allowing a better ROM and physical therapy participation postoperatively [26, 39, 40].

Whether a CIM TKA is associated with less blood transfusions, less intraoperative blood loss or a lower drop in haemoglobin postoperatively compared to OTS TKA remains controversial [26, 40, 41].

Anterior knee pain is a well-known problem after TKAs [42]. With CIM implants, the trochlea is designed to match the shape of the native patella and to maintain its native alignment, thus reducing patellar maltracking and the risk for secondary patellar resurfacing [16, 43].

The great variation in knee morphology among patients was seen in publications by Hirschmann et al. [8-10] assessing the association of limb alignment with the tibial and femoral joint line of 308 non-osteoarthritic patient knees. The authors determined eight phenotypes (i.e. $20 \%$ of all phenotypes found), which represent the coronal knee morphology of nearly $75 \%$ of the population. In contrast, the mechanical, anatomical and restricted kinematic alignment matched the phenotypes of only $5 \%, 20 \%$ and $51 \%$ of the population, respectively [10]. Thus, with the ORIGIN ${ }^{\circledR}$ alignment, which is based on the restricted phenotype alignment, the patient specific-knee morphology and limb alignment is better reproduced. Nevertheless, the comparison between CIM and 
OTS implants demonstrated that the former involved more physiological knee kinematics with greater weight bearing knee flexion and more posterior femoral rollback as well as greater axial rotation [44-46].

It is important to note that a measured resection technique bears the risk of underestimating an unequal and asymmetric laxity of the knee joint. This might lead to a less wellbalanced knee. Therefore, a more constrained TKA should be carefully considered for gross deformities. Similarly, a further aspect is the risk for the surgeon to rely heavily on the engineer's preoperative rather than on the intraoperative assessment [26].

The most relevant limitation of this study is the missing control group receiving standard OTS implants, thus these results can only be compared with the findings of other studies, which checked CIM versus OTS implants. The available literature, however, used different CIM implants and outcome measures. Second, the number of included patients is quite small and the follow-up is limited to 1 year, since the ORIGIN $^{\circledR}$ knee replacement system is only available since 2018. There is evidence showing an average time to revision below 1 year among patients with early knee implant failures [47]. Thus, despite the short follow-up time, preliminary results as presented in this study are of relevance. Third, CIM TKA are mostly performed in a private hospital setting and patient selection might be biased as most patients had an additional private insurance, which reflects a higher socioeconomic status. It is well known that these patients show increased satisfaction and better function. The demographics of the included patients do not fully overlap with the average patients undergoing TKA. Indeed, the former are younger and have a lower BMI, while the male gender is overrepresented [48-50].

\section{Conclusions}

This study demonstrated significant improvements in the KSS and specific PROMs 1 year after CIM TKA. With a satisfaction rate of $88 \%$ and no postoperative complications, this study suggests that TKA performed with a CIM implant is a safe and suitable option, which can yield good outcomes in terms of clinical outcome and PROMs at least in the short-term. Further studies should assess outcome scores and PROMs between CIM TKA and OTS TKA with different realignment strategies.

Author contributions C.S.M.: interpretation of data; writing of the original draft, preparation of the manuscript. M.T.H.: interpretation of data; writing of the original draft, preparation of the manuscript. N.V.: statistical analysis, review and editing of the manuscript. M.P.A.: performed the surgeries, conceptualisation, review and editing of the manuscript.

Funding Open Access funding provided by Universität Basel (Universitätsbibliothek Basel). No funding was obtained for this study.

\section{Declarations}

Conflict of interest M.P.A. and MTH are consultants for Symbios (Yverdon-les-Bains, Switzerland). C.S.M. and N.V. have no conflicts of interest.

Ethical approval This study was approved by the local ethics committee (reference: 2016-01777). The procedures used in this study adhere to the tenets of the Declaration of Helsinki.

Informed consent Written informed consent was obtained from all patients willing to participate.

Open Access This article is licensed under a Creative Commons Attribution 4.0 International License, which permits use, sharing, adaptation, distribution and reproduction in any medium or format, as long as you give appropriate credit to the original author(s) and the source, provide a link to the Creative Commons licence, and indicate if changes were made. The images or other third party material in this article are included in the article's Creative Commons licence, unless indicated otherwise in a credit line to the material. If material is not included in the article's Creative Commons licence and your intended use is not permitted by statutory regulation or exceeds the permitted use, you will need to obtain permission directly from the copyright holder. To view a copy of this licence, visit http://creativecommons.org/licenses/by/4.0/.

\section{References}

1. Hofmann S, Seitlinger G, Djahani O, Pietsch M (2011) The painful knee after TKA: a diagnostic algorithm for failure analysis. Knee Surg Sports Traumatol Arthrosc 19:1442. https://doi.org/ 10.1007/s00167-011-1634-6

2. Scott CEH, Howie CR, MacDonald D, Biant LC (2010) Predicting dissatisfaction following total knee replacement. J Bone Joint Surg Br 92-B:1253-1258. https://doi.org/10.1302/0301-620X. 92B9.24394

3. Brander V, Stulberg SD, Adams A et al (2003) Ranawat award paper: predicting total knee replacement pain: a prospective, observational study. Clin Orthop Relat Res 416:27-36. https:// doi.org/10.1097/01.blo.0000092983.12414.e9

4. Bourne RB, Chesworth BM, Davis AM et al (2010) Patient satisfaction after total knee arthroplasty: who is satisfied and who is not? Clin Orthop Relat Res 468:57-63. https://doi.org/10.1007/ s11999-009-1119-9

5. Baker PN, van der Meulen JH, Lewsey J et al (2007) The role of pain and function in determining patient satisfaction after total knee replacement. Data from the National Joint Registry for England and Wales. J Bone Joint Surg Br 89:893-900. https://doi.org/ 10.1302/0301-620X.89B7.19091

6. Vince KG, Abdeen A, Sugimori T (2006) The unstable total knee arthroplasty: causes and cures. J Arthroplasty 21:44-49. https:// doi.org/10.1016/j.arth.2006.02.101

7. Sharkey PF, Hozack WJ, Rothman RH et al (2002) Why are total knee arthroplasties failing today? Clin Orthop Relat Res 404:7-13

8. Hirschmann MT, Hess S, Behrend $\mathrm{H}$ et al (2019) Phenotyping of hip-knee-ankle angle in young non-osteoarthritic knees provides 
better understanding of native alignment variability. Knee Surg Sports Traumatol Arthrosc 27:1378-1384. https://doi.org/10. 1007/s00167-019-05507-1

9. Hirschmann MT, Moser LB, Amsler F et al (2019) Phenotyping the knee in young non-osteoarthritic knees shows a wide distribution of femoral and tibial coronal alignment. Knee Surg Sports Traumatol Arthrosc 27:1385-1393. https://doi.org/10.1007/ s00167-019-05508-0

10. Hirschmann MT, Moser LB, Amsler F et al (2019) Functional knee phenotypes: a novel classification for phenotyping the coronal lower limb alignment based on the native alignment in young non-osteoarthritic patients. Knee Surg Sports Traumatol Arthrosc 27:1394-1402. https://doi.org/10.1007/s00167-019-05509-z

11. Budhiparama NC, Lumban-Gaol I, Ifran NN et al (2020) Anthropometric measurement of Caucasian and Asian knees, mismatch with knee systems? Orthop J Sports Med 8:2325967120S00104. https://doi.org/10.1177/2325967120\$00104

12. Yue B, Varadarajan KM, Ai S et al (2011) Differences of knee anthropometry between Chinese and White men and women. J Arthroplasty 26:124-130. https://doi.org/10.1016/j.arth.2009.11. 020

13. Ha C-W, Na S-E (2012) The correctness of fit of current total knee prostheses compared with intra-operative anthropometric measurements in Korean knees. J Bone Joint Surg Br 94-B:638-641. https://doi.org/10.1302/0301-620X.94B5.28824

14. Henckel J, Richards R, Lozhkin K, et al (2006) Very low-dose computed tomography for planning and outcome measurement in knee replacement. J Bone Joint Surg Br 88-B:1513-1518. https:// doi.org/10.1302/0301-620X.88B11.17986

15. Bonnin MP, Beckers L, Leon A et al (2020) Custom total knee arthroplasty facilitates restoration of constitutional coronal alignment. Knee Surg Sports Traumatol Arthrosc. https://doi.org/10. 1007/s00167-020-06153-8

16. Sappey-Marinier E, Tibesku C, Selmi TAS, Bonnin M (2020) Custom total knee arthroplasty. In: Rivière C, Vendittoli P-A (eds) Personalized hip and knee joint replacement. Springer, Cham, pp 255-264

17. Arnold MP, Friederich NF, Widmer H, Müller W (1999) Lateraler Zugang zum Kniegelenk mit Osteotomie der Tuberositas tibiae. Operat Orthop Traumatol 11:223-232. https://doi.org/10.1007/ BF02593984

18. Vogel N, Rychen T, Kaelin R, Arnold MP (2020) Patient-reported outcome measures (PROMs) following knee arthroplasty: a prospective cohort study protocol. BMJ Open. https://doi.org/10. 1136/bmjopen-2020-040811

19. Scuderi GR, Bourne RB, Noble PC et al (2012) The new knee society knee scoring system. Clin Orthop Relat Res 470:3-19. https://doi.org/10.1007/s11999-011-2135-0

20. Roos EM, Lohmander LS (2003) The Knee injury and Osteoarthritis Outcome Score (KOOS): from joint injury to osteoarthritis. Health Qual Life Outcomes 1:64. https://doi.org/10.1186/ 1477-7525-1-64

21. Behrend H, Giesinger K, Giesinger JM, Kuster MS (2012) The "forgotten joint" as the ultimate goal in joint arthroplasty: validation of a new patient-reported outcome measure. J Arthroplasty 27:430-436.e1. https://doi.org/10.1016/j.arth.2011.06.035

22. EuroQol Group (1990) EuroQol - a new facility for the measurement of health-related quality of life. Health Policy 16:199-208. https://doi.org/10.1016/0168-8510(90)90421-9

23. Wheatley B, Nappo K, Fisch J et al (2019) Early outcomes of patient-specific posterior stabilized total knee arthroplasty implants. J Orthop 16:14-18. https://doi.org/10.1016/j.jor.2018. 11.003

24. Reimann P, Brucker M, Arbab D, Lüring C (2019) Patient satisfaction - a comparison between patient-specific implants and conventional total knee arthroplasty. J Orthop 16:273-277. https:// doi.org/10.1016/j.jor.2019.03.020

25. White PB, Ranawat AS (2016) Patient-specific total knees demonstrate a higher manipulation rate compared to "off-the-shelf implants." J Arthroplasty 31:107-111. https://doi.org/10.1016/j. arth.2015.07.041

26. Schwarzkopf R, Brodsky M, Garcia GA, Gomoll AH (2015) Surgical and functional outcomes in patients undergoing total knee replacement with patient-specific implants compared with "offthe-shelf” implants. Orthop J Sports Med 3:2325967115590379. https://doi.org/10.1177/2325967115590379

27. Kosse NM, Heesterbeek PJC, Schimmel JJP et al (2018) Stability and alignment do not improve by using patient-specific instrumentation in total knee arthroplasty: a randomized controlled trial. Knee Surg Sports Traumatol Arthrosc 26:1792-1799. https://doi. org/10.1007/s00167-017-4792-3

28. Maus U, Marques CJ, Scheunemann D et al (2018) No improvement in reducing outliers in coronal axis alignment with patientspecific instrumentation. Knee Surg Sports Traumatol Arthrosc 26:2788-2796. https://doi.org/10.1007/s00167-017-4741-1

29. Sassoon A, Nam D, Nunley R, Barrack R (2015) Systematic review of patient-specific instrumentation in total knee arthroplasty: new but not improved. Clin Orthop Relat Res 473:151158. https://doi.org/10.1007/s11999-014-3804-6

30. Cavaignac E, Pailhé R, Laumond G et al (2015) Evaluation of the accuracy of patient-specific cutting blocks for total knee arthroplasty: a meta-analysis. Int Orthop 39:1541-1552. https://doi.org/ 10.1007/s00264-014-2549-x

31. Zahn RK, Graef F, Conrad JL et al (2020) Accuracy of tibial positioning in the frontal plane: a prospective study comparing conventional and innovative techniques in total knee arthroplasty. Arch Orthop Trauma Surg 140:793-800. https://doi.org/10.1007/ s00402-020-03389-4

32. Behrend H, Zdravkovic V, Bösch M, Hochreiter B (2019) No difference in joint awareness after TKA: a matched-pair analysis of a classic implant and its evolutional design. Knee Surg Sports Traumatol Arthrosc 27:2124-2129. https://doi.org/10. 1007/s00167-019-05407-4

33. Thienpont E, Opsomer G, Koninckx A, Houssiau F (2014) Joint awareness in different types of knee arthroplasty evaluated with the forgotten joint score. J Arthroplasty 29:48-51. https://doi. org/10.1016/j.arth.2013.04.024

34. Wautier D, Thienpont E (2017) Changes in anteroposterior stability and proprioception after different types of knee arthroplasty. Knee Surg Sports Traumatol Arthrosc 25:1792-1800. https://doi.org/10.1007/s00167-016-4038-9

35. Naal FD, Impellizzeri FM, Lenze U et al (2015) Clinical improvement and satisfaction after total joint replacement: a prospective 12-month evaluation on the patients' perspective. Qual Life Res 24:2917-2925. https://doi.org/10.1007/ s11136-015-1042-3

36. Nielsen CS, Nebergall A, Huddleston J et al (2018) Medial overhang of the tibial component is associated with higher risk of inferior knee injury and osteoarthritis outcome score pain after knee replacement. J Arthroplasty 33:1394-1398. https://doi.org/ 10.1016/j.arth.2017.12.027

37. Dai Y, Scuderi GR, Penninger C et al (2014) Increased shape and size offerings of femoral components improve fit during total knee arthroplasty. Knee Surg Sports Traumatol Arthrosc 22:29312940. https://doi.org/10.1007/s00167-014-3163-6

38. Klasan A, Twiggs JG, Fritsch BA et al (2020) Correlation of tibial component size and rotation with outcomes after total knee arthroplasty. Arch Orthop Trauma Surg 140:1819-1824. https://doi.org/ 10.1007/s00402-020-03550-z

39. Namin AT, Jalali MS, Vahdat V et al (2019) Adoption of new medical technologies: the case of customized individually made 
knee implants. Value Health 22:423-430. https://doi.org/10. 1016/j.jval.2019.01.008

40. Culler SD, Martin GM, Swearingen A (2017) Comparison of adverse events rates and hospital cost between customized individually made implants and standard off-the-shelf implants for total knee arthroplasty. Arthroplast Today 3:257-263. https://doi. org/10.1016/j.artd.2017.05.001

41. Meheux CJ, Park KJ, Clyburn TA (2019) A retrospective study comparing a patient-specific design total knee arthroplasty with an off-the-shelf design: unexpected catastrophic failure seen in the early patient-specific design. J Am Acad Orthop Surg Glob Res Rev. https://doi.org/10.5435/JAAOSGlobal-D-19-00143

42. Petersen W, Rembitzki IV, Brüggemann G-P et al (2014) Anterior knee pain after total knee arthroplasty: a narrative review. Int Orthop 38:319-328. https://doi.org/10.1007/s00264-013-2081-4

43. Harrysson OLA, Hosni YA, Nayfeh JF (2007) Custom-designed orthopedic implants evaluated using finite element analysis of patient-specific computed tomography data: femoral-component case study. BMC Musculoskelet Disord 8:91. https://doi.org/10. 1186/1471-2474-8-91

44. Levengood GA, Dupee J (2018) Accuracy of coronal plane mechanical alignment in a customized, individually made total knee replacement with patient-specific instrumentation. J Knee Surg 31:792-796. https://doi.org/10.1055/s-0037-1608946

45. Patil S, Bunn A, Bugbee WD et al (2015) Patient-specific implants with custom cutting blocks better approximate natural knee kinematics than standard TKA without custom cutting blocks. Knee 22:624-629. https://doi.org/10.1016/j.knee.2015.08.002
46. Zeller IM, Sharma A, Kurtz WB et al (2017) Customized versus patient-sized cruciate-retaining total knee arthroplasty: an in vivo kinematics study using mobile fluoroscopy. J Arthroplasty 32:1344-1350. https://doi.org/10.1016/j.arth.2016.09.034

47. Sharkey PF, Lichstein PM, Shen C et al (2014) Why are total knee arthroplasties failing today-has anything changed after 10 years? J Arthroplasty 29:1774-1778. https://doi.org/10.1016/j.arth.2013. 07.024

48. Singh JA, Lewallen DG (2014) Time trends in the characteristics of patients undergoing primary total knee arthroplasty. Arthritis Care Res (Hoboken) 66:897-906. https://doi.org/10.1002/acr. 22233

49. Hylkema TH, Stevens M, Van Beveren J et al (2017) Preoperative characteristics of working-age patients undergoing total knee arthroplasty. PLoS ONE 12:e0183550. https://doi.org/10.1371/ journal.pone. 0183550

50. Basques BA, Bell JA, Sershon RA, Della Valle CJ (2018) The influence of patient gender on morbidity following total hip or total knee arthroplasty. J Arthroplasty 33:345-349. https://doi. org/10.1016/j.arth.2017.09.014

Publisher's Note Springer Nature remains neutral with regard to jurisdictional claims in published maps and institutional affiliations. 